Article

\title{
Noninvasive and Real-Time Plasmon Waveguide Resonance Thermometry
}

\section{Pengfei Zhang ${ }^{1,2}$, Le Liu ${ }^{3}$, Yonghong He ${ }^{1,2, *}$, Yanfei Zhou ${ }^{1,2}$, Yanhong $\mathrm{Ji}^{4}$ and Hui Ma ${ }^{1,2}$}

1 Shenzhen Key Laboratory for Minimal Invasive Medical Technologies, Institute of optical imaging and sensing, Graduate School at Shenzhen, Tsinghua University, Shenzhen 518055, China; E-Mails: zhangpf14@mails.tsinghua.edu.cn (P.Z.); zhou-yf12@mails.tsinghua.edu.cn (Y.Z.); mahui@tsinghua.edu.cn (H.M.)

2 Department of Physics, Tsinghua University, Beijing 100084, China

3 Institute of Green Chemistry and Energy, Graduate School at Shenzhen, Tsinghua University, Shenzhen 518055, China; E-Mail: liu.le@sz.tsinghua.edu.cn

4 MOE Key Laboratory of Laser Life Science \& Institute of Laser Life Science, South China Normal University, Guangzhou 510631, China; E-Mail: jiyh@ @scnu.edu.cn

* Author to whom correspondence should be addressed; E-Mail: heyh@ sz.tsinghua.edu.cn; Tel.: +86-137-9830-6211; Fax: +86-755-2603-6051.

Academic Editors: Gary R. Pickrell and Daniel Homa

Received: 21 January 2015 / Accepted: 8 April 2015 / Published: 13 April 2015

\begin{abstract}
In this paper, the noninvasive and real-time plasmon waveguide resonance (PWR) thermometry is reported theoretically and demonstrated experimentally. Owing to the enhanced evanescent field and thermal shield effect of its dielectric layer, a PWR thermometer permits accurate temperature sensing and has a wide dynamic range. A temperature measurement sensitivity of $9.4 \times 10^{-3}{ }^{\circ} \mathrm{C}$ is achieved and the thermo optic coefficient nonlinearity is measured in the experiment. The measurement of water cooling processes distributed in one dimension reveals that a PWR thermometer allows real-time temperature sensing and has potential to be applied for thermal gradient analysis. Apart from this, the PWR thermometer has the advantages of low cost and simple structure, since our transduction scheme can be constructed with conventional optical components and commercial coating techniques.
\end{abstract}


Keywords: plasmon waveguide resonance; thermometry; thermo optic effect; optical temperature sensor

\section{Introduction}

Temperature sensing is of importance in several fields [1], such as the microfluidic research [2], molecular interaction analysis [3] and clinical medicine [4]. Owing to the attractive advantages of being pollution free, having electric immunity and being of a compact size, the optical temperature sensors were studied and constructed by employing fluorescence dye [5], interferometer [6], microfiber knot resonator [7], optical microspheres [8], whispering-gallery mode dielectric resonator [9], Goos-Hänchen effect [10] and surface plasmon resonance (SPR) techniques [11]. The SPR thermometers permit noninvasive and real-time temperature sensing and have a simple sensor structure [11-13]. They have broad application prospect with the aim to provide an economical and convenient thermometer module based on the fact that the SPR sensors have been widely used for label-free refractive index (RI) related measurements [3,14-17]. Using the evanescent field of the surface plasmons, which propagate along the interface of two materials with real dielectric constants of opposite signs, the SPR thermometers probe the thermo optics (TO) coefficients of the materials for temperature sensing [11]. In the past decade, the temperature effect on the SPR sensors was studied at the bare metal film [12,18], metal-semiconductor interface [19], gold-water interface [20] and nanoparticles-dielectric interface [21]. SPR thermometers were constructed for transient thermal field analysis [22], microfluidic thermometry [13], in situ thermometry [23] and thermal gradient analysis [24]. However, because of the background TO effect of metal film, the sensitivity of the SPR thermometers is usually limited to the level of $0.1{ }^{\circ} \mathrm{C}$, which is achieved by using comprehensive analyses to measure the intrinsic temperature sensitivity [23]. The dynamic range is usually limited to about $10{ }^{\circ} \mathrm{C}$ achieved by using the angle interrogation [24] that has the widest dynamic range among four types of interrogations of the SPR sensors [25].

One effective method to improve the performance of SPR sensors is coating a dielectric layer on the metal film to enhance the evanescent field [26,27]. When the dielectric layer has both appropriate RI and thickness, the surface plasmons are coupled to the waveguide mode of the dielectric layer to construct the plasmon waveguide resonance (PWR) sensor, which is another kind of surface plasmon based optical sensors [28]. It has been pointed out that the performance of the PWR sensors can exceed that of the SPR sensors owing to enhancement of evanescent field and sharp reflectance spectrum in RI sensing applications $[17,27,29]$. Due to the small thermal conductivity of dielectric layers, the heat caused by wave propagation at the metal dielectric interface has less influence on the fluid RI in the PWR sensors [29]. Considering these advantages and the fact that the dielectric layer can protect the metal film against mechanical or chemical deterioration [30], we assume that the dielectric layer may have the ability to play the role of thermal shield of the metal film, which can improve the sensitivity and dynamic range of SPR based thermometers.

In this article, we report the PWR thermometer for noninvasive and real-time temperature sensing. The characteristics of the SPR and PWR sensors are compared, and the thermometry principles of the SPR and PWR sensors are analyzed. In a next step, the TO coefficient and cooling process of water are 
measured to test the system characteristics in the temperature measurement. In these experiments, we demonstrate that the PWR thermometer does not only have the advantages of the SPR thermometers, but also permits accurate temperature sensing in a wide temperature range because it can be free of background thermo optic effect of the sensing structure and has an enhanced evanescent field.

\section{Experimental Section}

\subsection{Design Consideration}

The well-known Kretschmann's analysis [31] is employed in this study. A gold (Au) film, which is commonly used in the SPR sensors [3], is employed as the surface plasmon active layer. A magnesium fluoride $\left(\mathrm{MgF}_{2}\right)$ film, which is easy to prepare and does not need an adhesion layer with a Au film [27], is employed as the dielectric layer. Considering that the transverse magnetic (TM) polarization has a large sensitivity to the RI variations [32] and better performance than the transverse electric (TE) polarization in the RI sensing applications [27,29], the performance of the PWR thermometer in the TM mode is the focus of this study. Using the multiple reflectance theory and Fresnel formula, the total reflectance in the TM mode can be calculated with:

$$
\begin{gathered}
R=\left|\frac{r_{12}+r_{2 \ldots k} \exp \left(2 i \beta_{2}\right)}{1+r_{12} r_{2 \ldots k} \exp \left(2 i \beta_{2}\right)}\right|^{2}, r_{2 \ldots k}=\frac{r_{23}+r_{3 \ldots k} \exp \left(2 i \beta_{3}\right)}{1+r_{23} r_{3 \ldots k} \exp \left(2 i \beta_{3}\right)} \\
r_{i, \mathrm{j}}=\frac{X_{i}-X_{j}}{X_{i}+X_{j}}, X_{i}=\frac{\varepsilon_{i}}{k_{i z}}
\end{gathered}
$$

where $k$ is the total layer number, $\varepsilon_{j}$ and $d_{j}$ are the dielectric constant and thickness of $j$ th layer, respectively, $\omega$ and $\theta$ are the angular frequency and incident angle of the incident light, respectively, and $c$ is light speed in the vacuum [33,34].

In this study, we employ the symmetrical PWR (sPWR) structure, which is also called the symmetrical optical waveguide structure [35] that has been used for the RI sensing applications [17]. As shown in Figure 1a, another $\mathrm{MgF}_{2}$ film is sandwiched between the prism and gold film in the sPWR structure instead of the adhesion layer, which is usually the chromium $(\mathrm{Cr})$ film (in this study) or titanium film used in the conventional PWR (cPWR) structure for available gold film firmness [27,29]. The angular reflectance spectra of the SPR, cPWR and sPWR sensors measuring the water are calculated and shown in Figure 1b. Combined with the method described by Hansen [36], the electric field strength distributions of the evanescent fields inside the water of the three structures are calculated and shown in Figure 1c. It can be seen from Figure 1 that the sPWR and cPWR sensors have similar evanescent field penetration depths and reflectance spectra, which are both longer and sharper than those of the SPR sensor. However, the angular reflectance spectrum of sPWR sensor has a higher extinction ratio than that of cPWR sensor, which can be duet to two reasons. One reason is that the long-range surface plasmon, which has a lower energy loss in the Au layer [37], is excited and coupled owing to the symmetrical condition [35]. The other reason is that the dielectric $\left(\mathrm{MgF}_{2}\right)$ layer has a smaller light absorption coefficient compared with the metal adhesion layer. Considering these facts and the good performance in the RI sensing applications [17], the sPWR sensing structure is employed in this study to construct the PWR thermometer. 


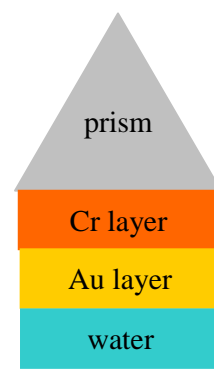

SPR

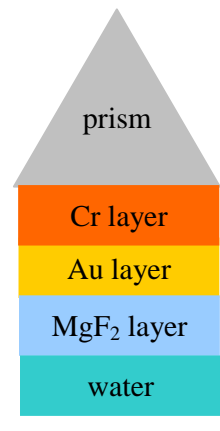

cPWR

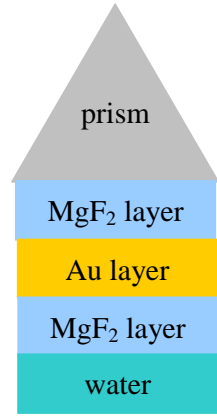

SPWR

(a)

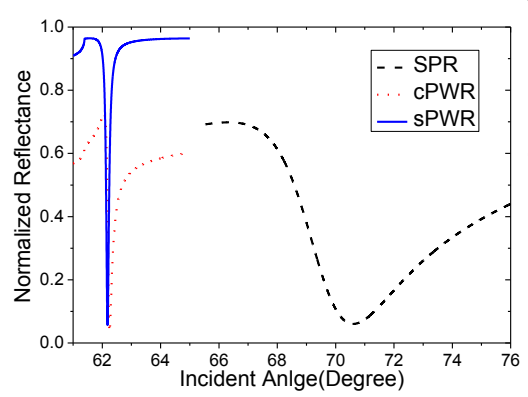

(b)

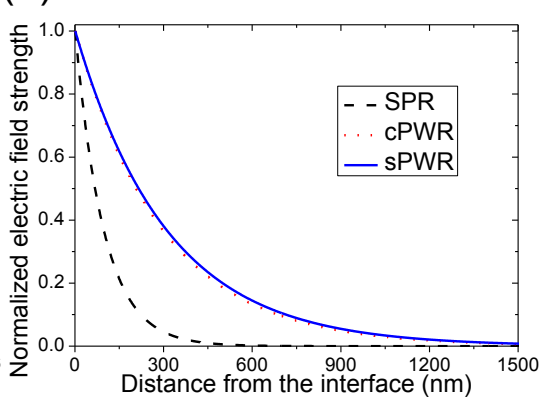

(c)

Figure 1. (a) Schematics of the surface plasmon resonance (SPR), conventional plasmon waveguide resonance (PWR) (cPWR), symmetrical PWR (sPWR) structures; (b) Angular reflectance spectra measuring water; (c) Normalized electric field strengths of the evanescent fields inside the water are plotted against the distance from the film/water interface. The refractive indices of the prism, $\mathrm{Cr}, \mathrm{Au}, \mathrm{MgF}_{2}$ and water we used are 1.515, $3.44+4.34 \mathrm{i}$, $0.13+3.65 \mathrm{i}, 1.38$ and 1.33. The thicknesses of $\mathrm{Cr}, \mathrm{Au}$ and $\mathrm{MgF}_{2}$ layers on contact with the water are $3 \mathrm{~nm}, 40 \mathrm{~nm}$, and $800 \mathrm{~nm}$. The $\mathrm{MgF}_{2}$ layer sandwiched between the prism and gold film in the sPWR structure is $500 \mathrm{~nm}$. The incident wavelength is $632.8 \mathrm{~nm}$.

It is known that the waveguide sensors can also be constructed without the surface plasmon active layer, namely the metal film, which is configured in the photonic mode based on the attenuated total reflection. In this experiment, this type of sensor can be constructed using some materials with high RI, such as $\mathrm{Si}_{3} \mathrm{~N}_{4}$ and $\mathrm{TiO}_{2}$. But the SPR and PWR sensors have distinct advantages in the measurement due to the sharper resonance dip and the fact that the spectral analysis can be easily used in the data processing of their signals, so the waveguide sensors using plasmonic mode, namely the PWR sensors are employed in this study.

The principles of SPR and PWR thermometers are similar regarding the measurement of the temperature dependent RI variation of samples within the evanescent field [11]. The relation between RI $n$ and relative permittivity $\varepsilon_{r}$ for the dielectric can be shown as [38]:

$$
n=\sqrt{\varepsilon_{r}}
$$

The relation between electronic polarizability $\alpha$ and relative permittivity $\varepsilon_{r}$ is:

$$
\alpha=\varepsilon_{0}\left(\varepsilon_{r}-1\right)
$$

where $\varepsilon_{0}$ is the vacuum permittivity [39]. The electronic polarizability of dielectric molecules usually varies with the temperature [40], which leads to a temperature dependent RI variation, namely the 
so-called TO effect. Hence the temperature of materials can be measured by using a RI sensor. The relation between RI $n$ and the temperature $T$ of the water, which is used as the sample in this study, is shown as:

$$
\begin{gathered}
\frac{n^{2}-1}{n^{2}+2} \frac{1}{\rho^{*}}=a_{0}+a_{1} \rho^{*}+a_{2} T^{*}+a_{3} \lambda^{* 2} T^{*}+a_{4} / \lambda^{* 2} \\
+\frac{a_{5}}{\lambda^{* 2}-\lambda_{U V}^{* 2}}+\frac{a_{6}}{\lambda^{* 2}-\lambda_{I R}^{* 2}}+a_{7} \rho^{* 2} \\
\rho^{*}=\frac{\rho}{\rho_{0}}, \lambda^{*}=\frac{\lambda}{\lambda_{0}}, T^{*}=\frac{T}{T_{0}}
\end{gathered}
$$

where $\rho$ is the density, $\lambda$ is the electromagnetic wavelength, $T$ is the absolute temperature, and $\rho_{0}, \lambda_{0}$, $\lambda_{U V}, \lambda_{I R}, T_{0}, a_{0}, a_{1}, a_{2}, a_{3}, a_{4}, a_{5}, a_{6}, a_{7}$ are constants [41]. Using Equations (5) and (6), the TO coefficient of water can be calculated to be in the order of $10^{-4} \mathrm{RIU} /{ }^{\circ} \mathrm{C}$.

In the SPR thermometer, the TO effect of an optical glass prism with the TO coefficients in the order of $10^{-6} \mathrm{RIU} /{ }^{\circ} \mathrm{C}$ can be neglected $[11,24]$. But the TO effect of the Au film, which is in contact with the samples and a good thermal conductor, cannot be neglected. The dielectric constant $\varepsilon$ of the Au film can be calculated with the Drude model:

$$
\varepsilon=\left(n_{r}+i n_{i}\right)^{2}=1-\frac{\omega_{p}^{2}}{\omega\left(\omega+i \omega_{c}\right)}
$$

where $n_{r}$ and $n_{i}$ are real and imaginary parts of RI, $\omega$ is the angular frequency of the incident light, and $\omega_{p}$ and $\omega_{c}$ are plasmon frequency and collision frequency of the electrons, respectively. The plasmon frequency can be calculated using:

$$
\omega_{p}(T)=\omega_{p}\left(T_{0}\right)\left[1+3 \gamma\left(T-T_{0}\right)\right]^{-\frac{1}{2}}
$$

where $\omega_{p}\left(T_{0}\right)$ is the plasmon frequency at a reference temperature $T_{0}$, and $\gamma$ is the thermal linear expansion coefficient [42]. In the metal film, the electron-phonon and electron-electron scattering processes contribute to the temperature dependent RI variation and can be estimated with:

$$
\omega_{c}=\omega_{0}\left[\frac{2}{5}+\frac{4 T^{5}}{\theta_{D}^{5}} \int_{0}^{\theta_{D} / T} \frac{z^{4}}{e^{z}-1} d z\right]+\frac{\pi^{4} \Gamma \Delta}{6 h E_{F}}\left[\left(k_{B} T\right)^{2}+\left(h \omega / 4 \pi^{2}\right)^{2}\right]
$$

where $\Gamma$ is the Fermi surface average of scattering probability, $\Delta$ is the fractional Umklapp scattering, $\theta$ is the Debye temperature, $E_{F}$ is the Fermi energy, $h$ is the Planck constant and $k_{B}$ is the Boltzmann constant $[43,44]$. Based on these equations, the TO coefficient of the Au film can be calculated as $d n_{r} / d T \approx 3.4 \times 10^{-4} \mathrm{RIU} /{ }^{\circ} \mathrm{C}$ and $d n_{i} / d T \approx-1.4 \times 10^{-4} \mathrm{RIU} /{ }^{\circ} \mathrm{C}$. They are in the same order with the TO coefficients of water. In a next step, the negative effect of the background TO effect of the Au film on the measurement accuracy of SPR thermometers is estimated. The propagation constant of the evanescent wave $k_{e}$ can be given by the standard phase matching condition

$$
k_{e}=k_{i n c} n_{p} \sin \theta
$$

where $k_{i n c}$ is propagation constant of the incident light wave, $n_{p}$ is the RI of the prism and $\theta$ is the incident angle. The evanescent wavelength in the SPR configuration shown in the Figure 1 can be 
estimated to be approximately $440 \mathrm{~nm}$. At this wavelength, the water TO coefficient can be estimated to be approximately $1.4 \times 10^{-4} \mathrm{RIU} /{ }^{\circ} \mathrm{C}$ when the temperature varies from $30{ }^{\circ} \mathrm{C}$ to $40{ }^{\circ} \mathrm{C}$ using Equations (5) and (6). Based on these considerations, the SPR angular reflectance spectra are calculated and shown in Figure $2 \mathrm{a}$ where the Au layer temperature varies from $30{ }^{\circ} \mathrm{C}$ to $40{ }^{\circ} \mathrm{C}$ and Figure $2 \mathrm{~b}$ where the water temperature varies from $30{ }^{\circ} \mathrm{C}$ to $40{ }^{\circ} \mathrm{C}$.

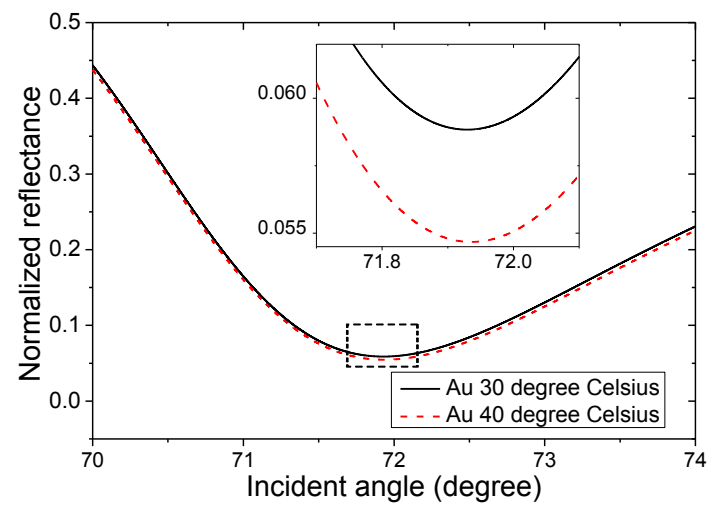

(a)

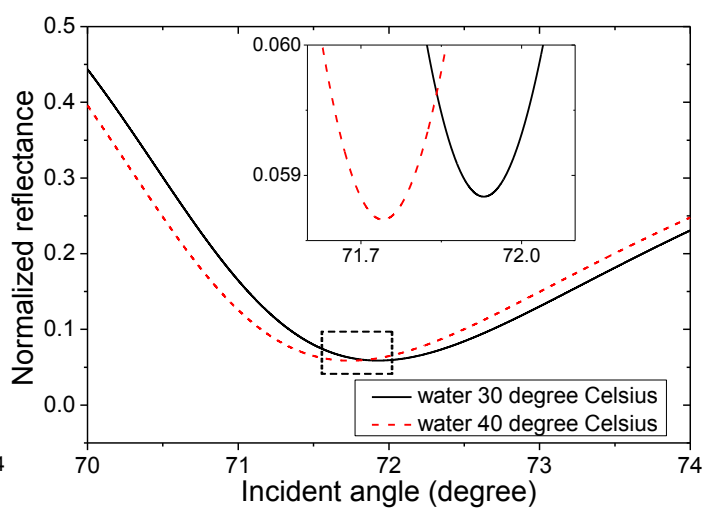

(b)

Figure 2. (a) Calculated SPR reflectance spectra when the temperature of the Au layer varies from $30{ }^{\circ} \mathrm{C}$ (the black solid line) to $40{ }^{\circ} \mathrm{C}$ (the red dash line) while the water temperature is maintained at $30{ }^{\circ} \mathrm{C}$; (b) Calculated SPR reflectance spectra when the water temperature varies from $30{ }^{\circ} \mathrm{C}$ (the black solid line) to $40^{\circ} \mathrm{C}$ (the red dash line) while the temperature of the Au layer is maintained at $30^{\circ} \mathrm{C}$. (Inset) the enlargement of the marked zones. At the temperature of $30{ }^{\circ} \mathrm{C}$, the refractive indices of the Au layer and water are $0.1324+3.6544 \mathrm{i}$ and $1.3403 \mathrm{RIU}$, respectively. At the temperature of $40^{\circ} \mathrm{C}$, the refractive indices of the $\mathrm{Au}$ layer and water are $0.1358+3.6530 \mathrm{i}$ and $1.3389 \mathrm{RIU}$, respectively. The refractive indices of the prism and $\mathrm{Cr}$ are 1.515 and $3.44+4.34 \mathrm{i}$. The thicknesses of the $\mathrm{Cr}$ and Au layers are $3 \mathrm{~nm}$ and $40 \mathrm{~nm}$, respectively. The incident wavelength is $632.8 \mathrm{~nm}$.

It can be seen from Figure 2 that the resonance angle, which is the incident angle affording the minimum reflectance intensity, shifts from $71.9295^{\circ}$ to $71.9359^{\circ}$ and the variation is $0.0064^{\circ}$ when only varying the Au layer temperature, and from $71.9295^{\circ}$ to $71.7410^{\circ}$ and the variation is $0.1885^{\circ}$ when only varying the water temperature. The temperature rise of the Au layer leads to the resonance angle shifting to a higher value, and the water temperature rise leads to the resonance angle shifting to a lower value. It can be seen that the resonance angle variation caused by the background TO effect of the Au layer is almost one-thirtieth of the variation caused by the water temperature variation. This has a deteriorating effect on the temperature measurement accuracy of the SPR thermometers, especially when used for measuring the temperature varying in a wide range.

In the PWR thermometer, the $\mathrm{MgF}_{2}$ layer is in contact with the water. The thermal conductivity coefficient, specific heat capacity and density of the $\mathrm{MgF}_{2}$ are $0.035 \mathrm{cal} /\left(\mathrm{s} \cdot \mathrm{cm} \cdot{ }^{\circ} \mathrm{C}\right)$ [45], $1020 \mathrm{~J} /\left(\mathrm{kg} \cdot{ }^{\circ} \mathrm{C}\right)$ and $3180 \mathrm{~kg} / \mathrm{m}^{3}$ [46], respectively, and the $\mathrm{MgF}_{2}$ has a TO coefficient of approximately $1 \times 10^{-6} \mathrm{RIU} /{ }^{\circ} \mathrm{C}$ [47], which is in the same order with that of optical glass and much lower than that of the Au film. Although the $\mathrm{MgF}_{2}$ layer is not a good thermal conductor and has a large specific heat capacity, which may cause the film temperature fluctuations of the SPWR sensing structure smaller than 
those of the SPR sensing structure, we analyze the worst case where the sPWR sensing structure has the same film temperature variation as the water. Using Equation (10), the evanescent wavelength in the sPWR configuration shown in the Figure 1 can be calculated to be approximately $473 \mathrm{~nm}$. At this wavelength, the water TO coefficient can be estimated to be approximately $1.4 \times 10^{-4} \mathrm{RIU} /{ }^{\circ} \mathrm{C}$ when the temperature varies from $30{ }^{\circ} \mathrm{C}$ to $40{ }^{\circ} \mathrm{C}$ using Equations (5) and (6). Based on these considerations, the angular reflectance spectra are calculated and shown in Figure $3 \mathrm{a}$ where the film temperature of the sPWR sensing structure varies from $30{ }^{\circ} \mathrm{C}$ to $40{ }^{\circ} \mathrm{C}$ while the water temperature is maintained at $30{ }^{\circ} \mathrm{C}$, and Figure $3 \mathrm{~b}$ where the water temperature varies from $30^{\circ} \mathrm{C}$ to $40^{\circ} \mathrm{C}$ while the film temperature of the sensing structure is maintained at $30^{\circ} \mathrm{C}$.

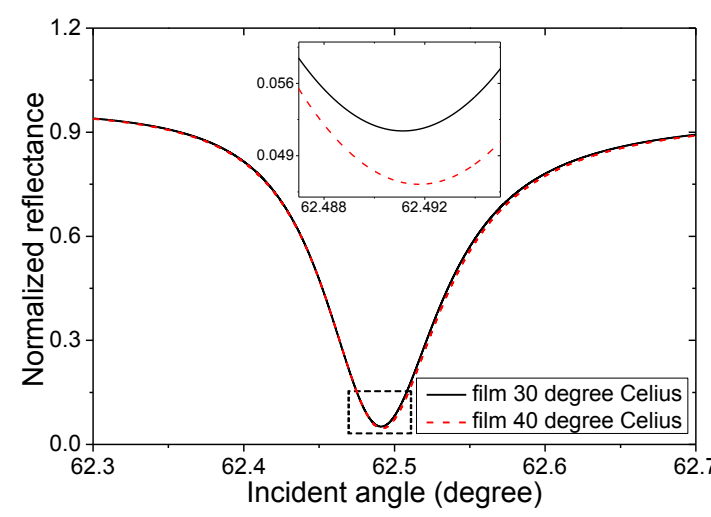

(a)

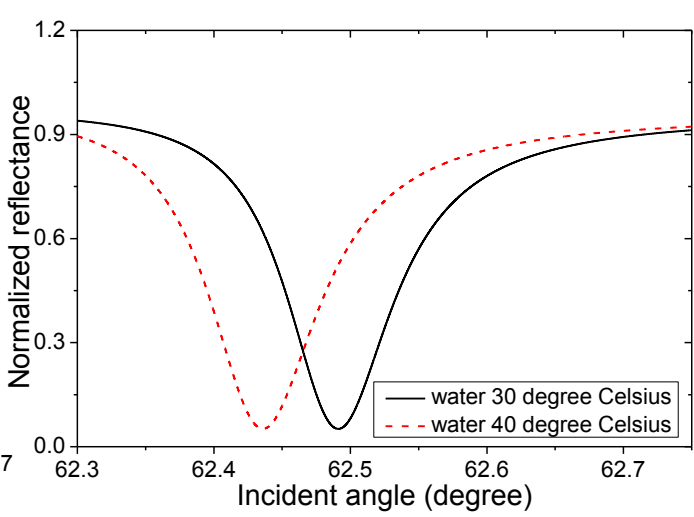

(b)

Figure 3. (a) Calculated sPWR reflectance spectra when the film temperature of the sensing structure varies from $30{ }^{\circ} \mathrm{C}$ (the black solid line) to $40{ }^{\circ} \mathrm{C}$ (the red dash line) while the water temperature is maintained at $30^{\circ} \mathrm{C}$; (b) Calculated sPWR reflectance spectra when the water temperature varies from $30{ }^{\circ} \mathrm{C}$ (the black solid line) to $40{ }^{\circ} \mathrm{C}$ (the red dash line) while the film temperature of sensing structure is maintained at $30^{\circ} \mathrm{C}$. (Inset) the enlargement of the marked zones. At the temperature of $30^{\circ} \mathrm{C}$, the refractive indices of the Au layer, $\mathrm{MgF}_{2}$ layer and water are $0.1324+3.6544 \mathrm{i}, 1.3804$ and $1.3381 \mathrm{RIU}$, respectively. At the temperature of $40{ }^{\circ} \mathrm{C}$, the refractive indices of $\mathrm{Au}$ layer, $\mathrm{MgF}_{2}$ layer and water are $0.1358+3.6530 \mathrm{i}, 1.38041$ and 1.3367 RIU, respectively. The RI of the prism is 1.515. The thicknesses of Au layer and $\mathrm{MgF}_{2}$ layer in contact with the water are $40 \mathrm{~nm}$ and $800 \mathrm{~nm}$, respectively. The $\mathrm{MgF}_{2}$ layer sandwiched between the prism and gold layer is $500 \mathrm{~nm}$. The incident wavelength is $632.8 \mathrm{~nm}$.

It can be seen from Figure 3 that the resonance angle, which is the incident angle affording the minimum reflectance intensity, shifts from $62.4911^{\circ}$ to $62.4917^{\circ}$ and the variation is $0.0006^{\circ}$ when only varying the film temperatures of sPWR sensing structure, and it shifts from $62.4911^{\circ}$ to $62.4359^{\circ}$ and the variation is $0.0552^{\circ}$ when only varying the water temperature. The temperature rise of the sensing structure leads to the resonance angle shifting to a higher value, and the water temperature rise leads to the resonance angle shifting to a lower value. It can be seen that the resonance angle variation caused by the background TO effect of the sensing structure is almost one percent of the variation caused by the water temperature variation. The negative effect of the background TO effect of sPWR sensing films is about five times smaller than that of the SPR sensing film. This indicates that the sPWR thermometer 
could suppress the negative effect of background TO effect significantly. The thermal expansion effect is usually the other interfering factor in the optical temperature sensing. However considering the fact that the linear thermal expansion coefficients of $\mathrm{Au}$ and $\mathrm{MgF}_{2}$ are both in the order of $10^{-5} /{ }^{\circ} \mathrm{C}[11,48]$, the film thickness variation caused by the thermal expansion effect can be neglected in both SPR and PWR thermometers. In summary, it is possible to construct a thermometer allowing accurate temperature sensing in a wide dynamic range using the PWR technique.

\subsection{Sensor Chips}

A polished BK7 glass substrate is firstly cleaned with a solution consisting of ethanol and diethyl ether in 1:1 ratio, then rinsed with deionized water (Milli-Q water) and dried with nitrogen. Then the substrate is sequentially coated with a $500 \mathrm{~nm} \mathrm{MgF}$ film, a $40 \mathrm{~nm}$ Au film and an $800 \mathrm{~nm} \mathrm{MgF}$ film to construct the sPWR sensor chip. The gold film is coated using magnetron sputtering with the film thickness measured by a quartz crystal oscillator thickness monitor. The substrate heating and bias voltage techniques are employed in the coating process to improve the Au film thickness uniformity and firmness [24]. The $\mathrm{MgF}_{2}$ film is deposited using evaporation coating with the film thickness measured by a step profiler. The $\mathrm{MgF}_{2}$ crystals are used as the evaporation materials and the weight is controlled for by the specific film thickness in the coating process [27]. In a next step, the sensor chip is placed on a BK7 prism with the RI matching oil (Cargille) to construct a sPWR sensor module.

\subsection{Experimental Setups}

A conventional one-dimensional imaging system is employed in this study as shown in Figure 4. The integrated light source (LS) employs a $25 \times$ objective lens to focus the light from a red light emitting diode with an electric power of $3 \mathrm{~W}$ on a pinhole. Then the light is collimated by the collimation lenses consisting of two convex lenses (L1, L3) and one concave lens (L2) before being filtered by a bandpass filter (BF, center wavelength $632.8 \mathrm{~nm}$, bandwidth $10 \mathrm{~nm}$, Thorlabs), and p-polarized by a linear glass polarizer (P). An aperture (A) is used to acquire a rectangular light spot. After passing through a cylindrical lens (focus length $40 \mathrm{~mm}$ ) with a vertical axis of symmetry, the light is focused to a narrow line on the sensing surface (SS) to construct an imaging channel. The sPWR module is configured in the Kretschmann manner with the attenuated total reflection method in this system. A polymethyl methacrylate flow cell (FC, $10 \times 3 \times 3 \mathrm{~mm}, 90 \mu \mathrm{L}$ ) is attached to the sensor chip for sample delivery to the sensing surface through the fluidic channel $(\mathrm{CH})$. The reflection light beams are recorded by a charge coupled device (CCD, $2580 \times 1912$ pixels, $3.4 \mu \mathrm{m}$ pixel size, ICX282AQ, Sony) for further data analysis in a personal computer (PC). The temperature control system is configured in the manner described in $[24,49]$. The sample continuously circulates through a programmable water bath (WP). A peristaltic pump (PUMP) circulates the water through the cell at a rate of about $10 \mathrm{~mL} / \mathrm{min}$. The temperature in the cell is measured by two digital thermocouple probes $(\mathrm{T} 1, \mathrm{~T} 2)$ with $0.1{ }^{\circ} \mathrm{C}$ resolution. 


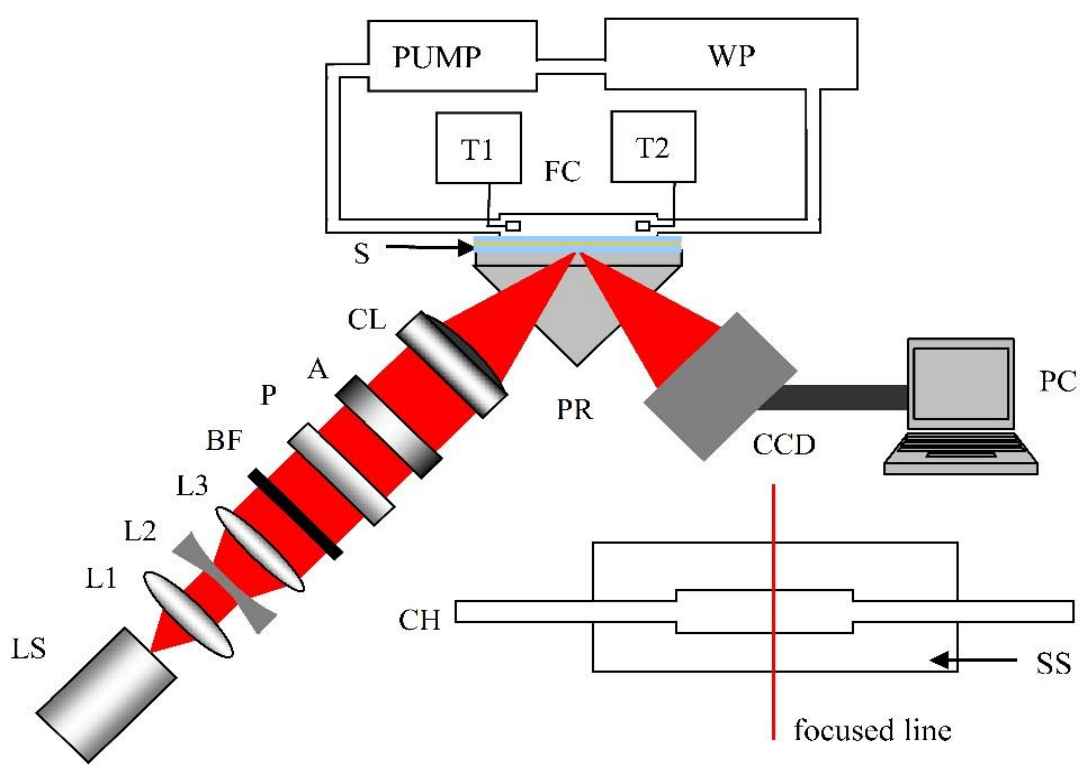

Figure 4. Schematic of the experimental system: LS, integrated light source; L1, convex lens; L2, concave lens; L3, convex lens; BF, bandpass filter; $\mathrm{P}$, linear glass polarizer; A, rectangular aperture; CL, cylindrical lens; PR, prism; S, sensor chip; FC, flow cell; CCD, charge coupled device; PC, personal computer. WP, programmable water path; PUMP, peristaltic pump; T1, T2, digital thermocouple probes; $\mathrm{CH}$, fluidic channel; SS, sensing surface.

In the experiment, the resonance angle is represented by the corresponding pixel position affording the minimum intensity in the captured image. The facula after passing the rectangular aperture forms a square with the size of $10 \times 10 \mathrm{~mm}^{2}$. Using the focus length of the cylindrical lens and considering the refraction effect of the prism, the angular range of the incident wedge light beam is approximately $8.13^{\circ}$. The reflectance light beam forms a rectangle with the size of approximately $52 \times 10 \mathrm{~mm}^{2}$ when captured by the CCD. Considering the pixel size, the angular resolution of the imaging system can be calculated to be $5.3 \times 10^{-4}$ degree/pixel. In this imaging system, the left area in the CCD sensing area, where the pixel number is represented by small values in the captured images, records the reflectance light beam with a large incident angle. This is due to the CCD characteristics.

\section{Results and Discussion}

\subsection{RI Test}

The deionized water and the glucose solutions with different indices are used to test the sensor response to the RI change. The relationship between RI $n$ and concentration $C$ of the glucose solution is $n=n$ (water) $+1.515 \times 10^{-4} \times C$, where $C$ is the concentration in grams per liter. The experimental temperature is approximately $27^{\circ} \mathrm{C}$ and the temperatures of the solutions are controlled to be $27^{\circ} \mathrm{C}$. The evanescent wavelength in the sPWR sensor can be estimated to be approximately $473 \mathrm{~nm}$ with Equation (10), so the water RI can be calculated to be 1.3384 using Equations (5) and (6). In this experiment, the resonance angle is represented by the corresponding pixel position affording the minimum intensity. To suppress the deviation caused by the response non-uniformity of the CCD, a 
hundred adjacent rows in one image are averaged to acquire the angular spectrum measuring the samples flowing through corresponding area. A ten order polynomial curve fitting method is applied to reduce the noise for determination of the pixel position of the resonance angle [50]. The water and the glucose solutions with concentrations of 1,2,3, 4, 5, 6 and $7 \mathrm{~g} / \mathrm{L}$ are measured serially. Fifty images are captured with an exposure time of $600 \mathrm{~ms}$ for each sample and the capture time interval is $5 \mathrm{~s}$.

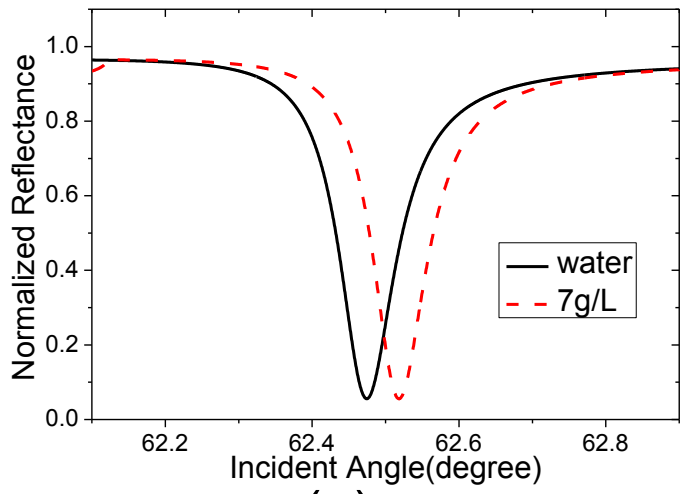

(a)

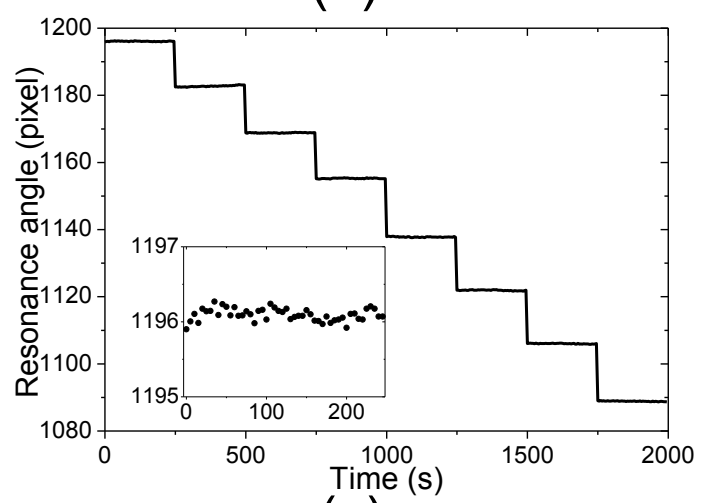

(c)

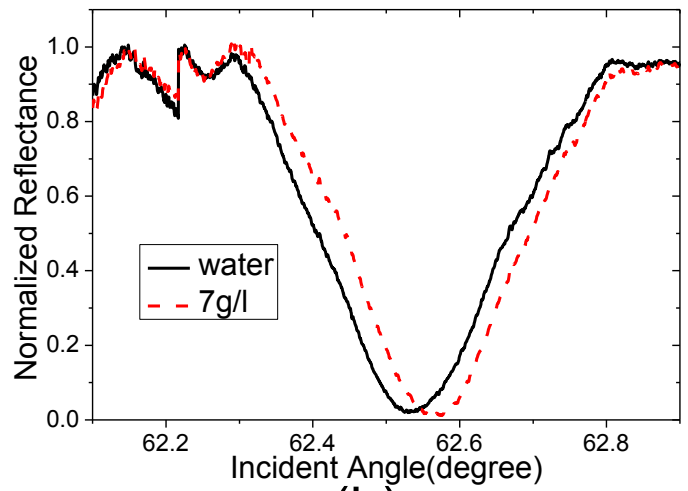

(b)

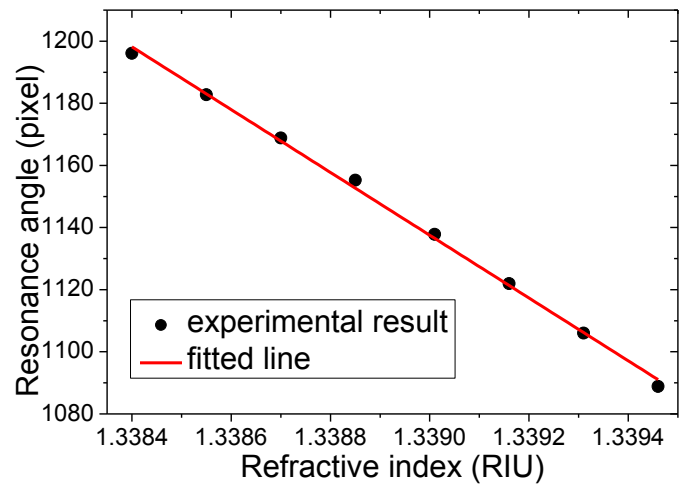

(d)

Figure 5. (a,b) Calculated, experimental angular reflectance spectra measuring the water and glucose solution with a concentration of $7 \mathrm{~g} / \mathrm{L} ;(\mathbf{c})$ Measured resonance angle as function of time, inset: enlargement of the function measuring the water at the time interval from $0 \mathrm{~s}$ to $250 \mathrm{~s}$; (d) Averaged measured resonance angle as function of the refractive index. The error bars representing the standard errors lie within the data characters.

Figure 5a,b shows the theoretical and experimental angular reflectance spectra measuring the water and glucose solution with a concentration of $7 \mathrm{~g} / \mathrm{L}$. The measured resonant angles as the function of time and averaged measured resonance angle as the function of RI are shown in Figure 5c,d, respectively. In this experimental setup, the pixel positions with larger values represent the smaller incident angle due to the system characteristics. The combined sensitivity factor $(C S F)$ [51] and RI resolution $\sigma$ [52] are employed to estimate sensor characteristics. These functions are shown as:

$$
C S F=S F \times \frac{R_{\max }-R_{\min }}{F W H M}, \sigma=\frac{\sigma_{S O}}{S F}, S F=\frac{\partial \theta}{\partial n}
$$

where $\theta$ is the incident angle, $n$ is the RI of sample, $R_{\max }$ and $R_{\min }$ are the maximum and minimum normalized reflectance, $F W H M$ is the full width at half maximum, $\sigma_{S O}$ is the standard deviation of sensor output, and $S F$ is the sensitivity factor. Based on these functions and Figure 5a,b, the theoretical 
and experimental CSFs of sPWR are approximately 381 and 206, respectively. It can be seen from Figure 5c,d that the sensor output response is linear for the RI and the sensitivity factor is $1.01 \times 10^{5}$ pixels/RIU, namely 53.5 degree/RIU. The standard deviation of the baseline created by the deionized water, whose RI is free of evaporation effects, is 0.082 pixel, so the RI resolution of this sPWR sensor can be calculated to be $7.9 \times 10^{-7}$ RIU. The performance of this sPWR sensor is compared with those of the cPWR sensor using an $\mathrm{Au}-\mathrm{MgF}_{2}$ structure [27], the cPWR sensor using an Au-Silica structure and the SPR sensor [29] at the incident wavelength of $632.8 \mathrm{~nm}$ in the TM mode as shown in Table 1. In these studies, similar experimental methods are employed without optimization for either data process algorithm or optical system, which employs the high quality area detector [35] or new technique such as the polarization interferometry [25].

Table 1. Comparison of the PWR and SPR sensors' characteristics at the incident wavelength of $632.8 \mathrm{~nm}$ in the transverse magnetic (TM) mode.

\begin{tabular}{cccc}
\hline Sensor & Theoretical CSF $\left(\right.$ RIU $\left.^{-1}\right)$ & $\begin{array}{c}\text { Experimental CSF } \\
\left(\text { RIU }^{-1}\right)\end{array}$ & RI Resolution (RIU) \\
\hline SPR & 14 & 12 & $3.2 \times 10^{-6}$ \\
Silica-cPWR & 37 & 38 & $2.3 \times 10^{-6}$ \\
$\mathrm{MgF}_{2}$-cPWR & 167 & 142 & $9.3 \times 10^{-7}$ \\
sPWR & 381 & 206 & $7.9 \times 10^{-7}$ \\
\hline
\end{tabular}

It can be seen that the RI resolution and theoretical and experimental CSFs of sPWR sensor are all better than those of SPR and cPWR sensors at the incident wavelength of $632.8 \mathrm{~nm}$. This enhancement can be due to the extinction ratio improvement of the angular reflectance spectrum and enhanced evanescent field, which can be supported by the theoretical predictions shown in Figure 1. It should be noticed that the difference between the theoretical and experimental CSFs of the sPWR sensor is larger than that between the cPWR and SPR sensors, which can be explained by in two ways. One reason is more the complicated sensing film structure of the sPWR sensor, so the matching relation is more difficult to be preserved in the practical applications due to film quality fluctuation in the coating process. The other reason is that both the $\mathrm{MgF}_{2}$ layers have large film thicknesses, so the negative effect of the film thickness errors in the coating process will be more significant. Considering these, the sPWR sensor still shows its advantage in the RI sensing application, whose RI resolution of $7.9 \times 10^{-7} \mathrm{RIU}$ is at the best level that can be provided by the SPR sensor under the incident wavelength of $632.8 \mathrm{~nm}$ predicted by Piliarik and Homola [52].

\subsection{Temperature Sensing}

The temperature variations of water, a material widely used for research and applications, are measured to test the system characteristics in the temperature sensing application. The water RI has little deviation from the linear correspondence to the temperature in a wide temperature range [41], but its response can be considered to be linear for the temperature in a narrow temperature range [13,24]. In this experiment, we firstly control the water temperature varying from $30{ }^{\circ} \mathrm{C}$ to $40{ }^{\circ} \mathrm{C}$ every $2{ }^{\circ} \mathrm{C}$ to test the measurement accuracy of this system, and then from $30{ }^{\circ} \mathrm{C}$ to $80{ }^{\circ} \mathrm{C}$ every $10{ }^{\circ} \mathrm{C}$ to demonstrate the TO coefficient nonlinearity of water. The room temperature is set to be $30{ }^{\circ} \mathrm{C}$ using the air conditioner. 
The sensor outputs measuring the water at the temperature of $30{ }^{\circ} \mathrm{C}$ are used as the baseline. Fifty images are captured with the exposure time of $600 \mathrm{~ms}$ for each temperature and the capture time interval is $5 \mathrm{~s}$.

Figure 6 a shows the measured resonant angles probing the water temperature varying from $30{ }^{\circ} \mathrm{C}$ to $40{ }^{\circ} \mathrm{C}$ every $2{ }^{\circ} \mathrm{C}$ as the function of time, and Figure $6 \mathrm{~b}$ shows the averaged measured resonance angle as the function of the water temperature. The experimental results and theoretical refractive indices of water in the temperature range from $30{ }^{\circ} \mathrm{C}$ to $80{ }^{\circ} \mathrm{C}$ every $10{ }^{\circ} \mathrm{C}$ are shown in Figure $6 \mathrm{c}$ and the experimental results as the function of the theoretical refractive indices are shown in Figure $6 \mathrm{~d}$.

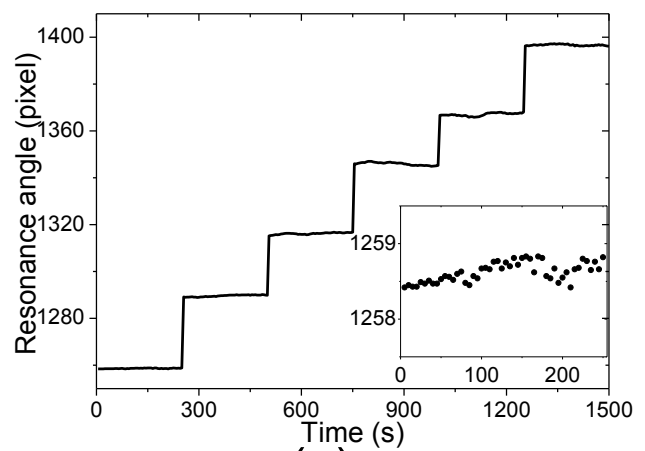

(a)

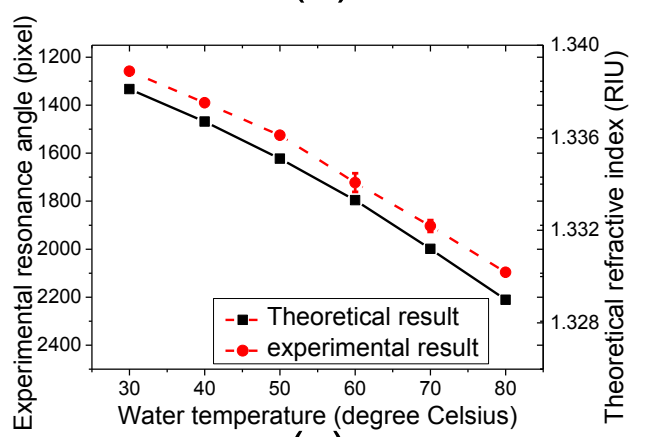

(c)

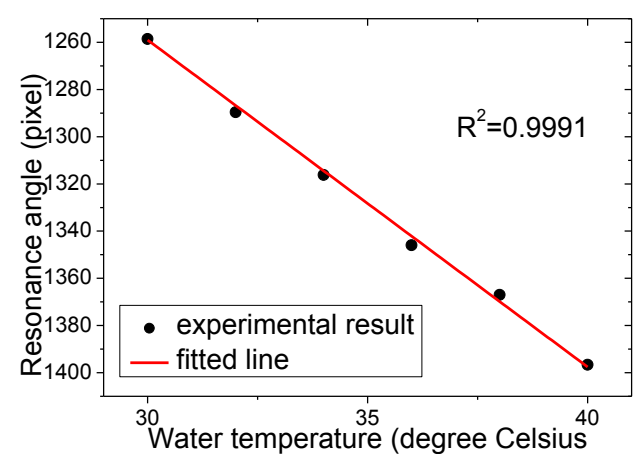

(b)

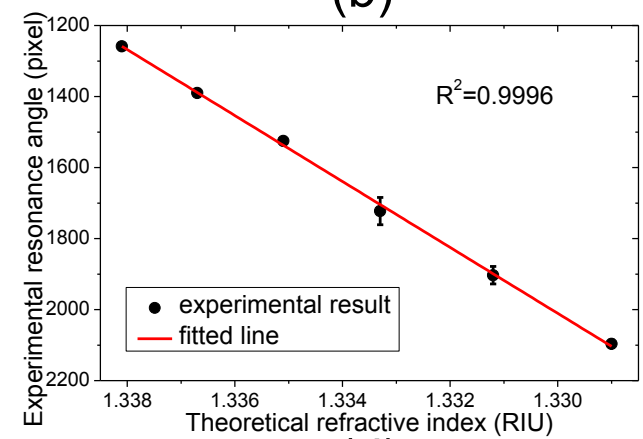

(d)

Figure 6. (a) Measured resonance angle as function of time, inset: enlargement of the function measuring the water at the temperature of $30{ }^{\circ} \mathrm{C}$ at the time interval from $0 \mathrm{~s}$ to $250 \mathrm{~s}$; (b) Averaged measured resonance angle as function of the water temperature. The error bars representing the standard errors lie within the data characters; (c) Comparison between experimental resonance angles and theoretical refractive indices. The error bars represent the standard errors; (d) Measured resonance angle as function of the water refractive index at different temperature. The error bars represent the standard errors.

It can be seen from Figure 6a that the pixel position of the resonance angle shifts to the large value with temperature rising, which means the resonance angle shifts to a small value in this system when the water RI decreases. This is in agreement with the experimental results shown in Figure 5. It can be seen from Figure $6 \mathrm{~b}$ that the sensor output responds linearly to the temperature, which means the water TO coefficient could be seen as a constant in this temperature range to estimate the thermometer characteristics in the temperature sensing applications. The measured TO coefficient in the temperature range from $30{ }^{\circ} \mathrm{C}$ to $40{ }^{\circ} \mathrm{C}$ is $13.86 \pm 0.19$ pixels $/{ }^{\circ} \mathrm{C}$. After converted the pixel shift to RI using the sensitivity factor obtained in the RI test, the TO coefficient of water in this temperature range can be calculated to be $(1.37 \pm 0.019) \times 10^{-4} \mathrm{RIU} /{ }^{\circ} \mathrm{C}$. This is close to the calculated values of 
$1.40 \times 10^{-4} \mathrm{RIU} /{ }^{\circ} \mathrm{C}$ for water using Equations (5) and (6) under the evanescent wavelength of $473 \mathrm{~nm}$ in this temperature range. The deviation may be because of the measurement errors and difference between the water quality in the experiment and calculations. In order to compare the temperature measurement sensitivity among various approaches, the temperature measurement sensitivity $\sigma_{T}$ is calculated according to

$$
\sigma_{T}=\frac{d T}{d p} \sigma_{p}
$$

where $p$ is the parameter being monitored during the measurement of the temperature variation, $T$ is the temperature, and $\sigma_{p}$ is the available parameter resolution of experimental system [12]. In this experiment, the pixel position shift is the monitored parameter and the parameter resolution is the standard error of the baseline [52]. Considering the standard error of 0.13 pixels for the baseline shown in the insert in Figure 6a, the temperature sensitivity of this system can be calculated to be $9.4 \times 10^{-3}{ }^{\circ} \mathrm{C}$, which is comparable to the recently reported high-resolution optical temperature sensor [10]. It can be seen that the standard error of sensor output in the temperature sensing is larger than that in the RI test, which should be caused by the noise of the temperature control system in the process of varying water temperature.

When the temperature range is wide, the water nonlinearity cannot be neglected, which can be seen from the theoretical predictions and reported experimental results achieved by using highly accurate methods [41]. It can be seen from Figure $6 \mathrm{c}$ that both the theoretical and experimental results show the water TO coefficient nonlinearity. Because of the small value of the TO coefficient nonlinearity and the limitation caused by the dynamic range or the background noise, the nonlinearity has not been reported in the experimental results of current SPR thermometers [12,13,22-24], and is hardly observed by other reported optical temperature sensors [5-10]. To demonstrate the feasibility of the PWR thermometry for accurate TO coefficient sensing in a wide temperature range, the experimental resonance angle is fitted linearly to the theoretical RI as shown in Figure $6 \mathrm{~d}$. It can be seen that the sensor setup responds to the temperature dependent RI variation linearly as it does in the RI test. This indicates that the PWR thermometer can measure the TO coefficient accurately so it can keep the measurement accuracy for the temperature sensing in the wide temperature range.

\subsection{Real Time Natural Cooling Process Measurement}

To demonstrate the feasibility of this system for real time temperature sensing, the natural cooling process of water is measured. The water at a temperature of $40{ }^{\circ} \mathrm{C}$ circulates through the flow cell continuously. Then we turn off the pump and the temperature control device in the water path. Considering the system response delay, we start to capture images at $10 \mathrm{~s}$ and stop at $600 \mathrm{~s}$. The capture time interval is $5 \mathrm{~s}$. The final temperature is $30.1^{\circ} \mathrm{C}$ measured by the thermocouple probe, and the room temperature is set to be $30{ }^{\circ} \mathrm{C}$ using the air conditioner. In this situation, the cooling process should follow Newton's cooling law:

$$
\begin{gathered}
\frac{d T}{d t}=-k(T-C) \\
T=C+\left(T_{0}-C\right) e^{-k t}
\end{gathered}
$$


where $T$ is the water temperature at the time $t, T_{0}$ is the initial temperature, $k$ is a constant, and $C$ is the room temperature. The cooling process in the area of a hundred rows measured in the above experiments, which is located in the middle area of the flow cell and marked by "area 2" in Figure 7a, is analyzed as shown in Figure 7d. For reference analysis, the areas of the same hundred rows located on the sides of this area, which are marked with "area 1" and "area 3" in Figure 7a, are also analyzed and shown in Figures $7 \mathrm{c}$ and e respectively. The cooling curves of water in three areas are shown by 3D waterfall plots in Figure $7 \mathrm{~b}$. The comparisons between the theoretical and experimental constant $k$ and initial temperature $T_{0}$ are shown in Figure 7f,g, respectively.

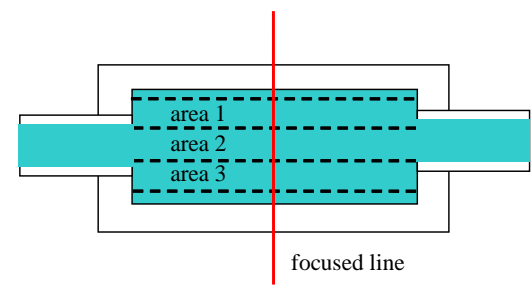

(a)

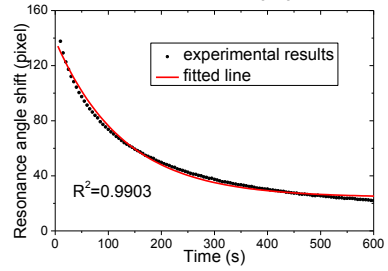

(c)

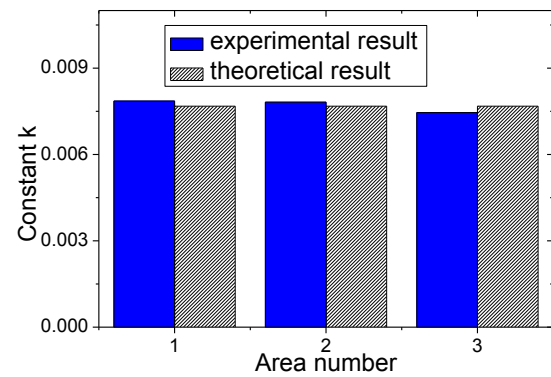

(f)

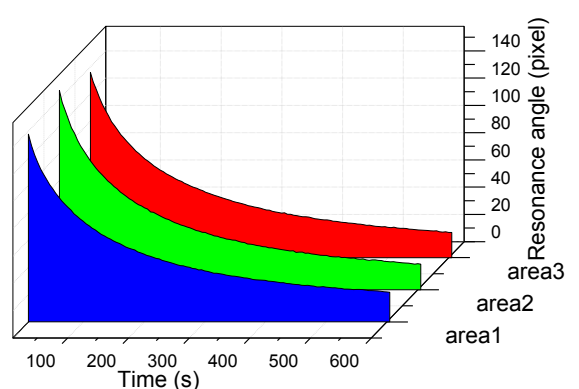

(b)

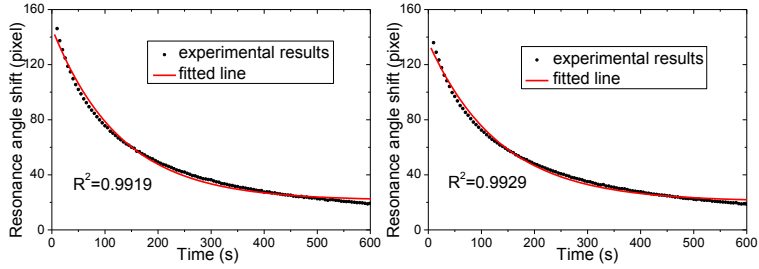

(e)

(d)

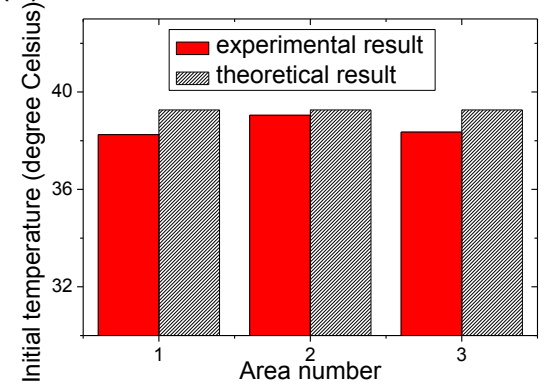

(g)

Figure 7 (a) Areas of interest marked with areas 1, 2, 3 are at the top, middle and bottom along the focused line in the cell respectively; (b) Waterfall plots of water cooling curves in the areas 1, 2, 3; (c-e) Experimental and fitted cooling curves of water cooling processes located in areas 1, 2, 3; (f) Comparison between the theoretical and experimental constants $k$; (g) Comparison between experimental and theoretical initial temperatures.

It can be seen from Figure 7c-e that the experimental results are all well fitted to Newton's cooling law. It also can be seen from Figure $7 \mathrm{f}$ that the constant $k$ in the three areas is similar and close to the theoretical results. This demonstrates that the cooling processes can be measured by this thermometer in real time. The deviations may be caused by the change of thermal conduction mode and timing error in the measurement and the coefficient effect for different sensing channels.

It can be seen from the Figure $7 \mathrm{~g}$ that the initial water temperatures in area 2 are slightly lower than the theoretical initial temperature of $39.26^{\circ} \mathrm{C}$ calculated by Equation (14), which should be caused by 
the timing error. It can also be seen that the initial temperature at side areas, namely areas 1 and 3 are lower. Apart from the timing error, this deviation also could be caused by three factors. Firstly, due to the water flowing into and out of the flow cell through the holes in the middle of the side walls in this system, the water temperature in the middle area is easier to be controlled to the given temperature. Secondly, side areas are closer to the environment so they have a better heat exchange, whereas the middle area is surrounded by water with a large specific heat capacity, so the water temperature is hardly influenced by the environment temperature variations. Thirdly, there may be residual air in the flow cell, which stays at the side areas more easily due to the buoyancy and viscous force, which also has a negative effect on the temperature control in the side areas. Despite these facts, this system shows the ability to monitor temperature variations in real time. Apart from this, the cooling processes in different areas are measured simultaneously in this experiment, which indicates that this thermometer has the potential to be used for thermal gradient analysis.

\section{Conclusions}

We report on PWR (plasmon waveguide resonance) thermometry in this paper. The sPWR (symmetrical PWR) sensor, a kind of PWR sensors, is employed to demonstrate the feasibility experimentally. Owing to the enhancement of the evanescent field and the improvement of the extinction ratio in the reflectance spectrum, the sPWR sensor can achieve a RI resolution of $7.9 \times 10^{-7}$ RIU. In the temperature sensing experiment, owing to the thermal shield effect of the dielectric layer and the improvement of the RI resolution, the PWR thermometer can achieve a temperature sensitivity of $9.4 \times 10^{-3}{ }^{\circ} \mathrm{C}$, which is comparable to the recently reported high resolution optical temperature sensor [7]. Additionally, the TO coefficient nonlinearity is clearly observed using this thermometer, and it is so small that it is hardly measured by current optical thermometers. This indicates that the PWR thermometer has a wide dynamic range, and it may improve the measurement accuracy of optical thermometers in noninvasive temperature sensing applications. Finally, the cooling process monitoring shows that this method has the ability to monitor temperature in real time and the potential to be applied for thermal gradient analysis. Using the convention optical components and commercial coating techniques, the PWR thermometer could be easily implemented in practical applications, as the system has a simple optical arrangement and a good compatibility with a variety of SPR platforms, which have been used in diverse fields. PWR thermometry may promote the applications of optical temperature sensors and simulate further studies of new methods for noninvasive and real-time thermometry using electromagnetic fields.

\section{Acknowledgements}

This research was made possible with the financial support from NSFC China $(61275188,61378089$, 61361160416), the 863 project, China, Shenzhen International cooperation project, and the Technology Development Program of Shenzhen City.

\section{Author Contributions}

Pengfei Zhang is the main author of this article, including the system design, experimental work and writing the paper. Le Liu and Yanfei Zhou have participated in the discussions and experiments. 
Yonghong He conceives the experiments. Yanhong Ji and Hui Ma review the theoretical analysis and experimental results.

\section{Conflicts of Interest}

The authors declare no conflict of interest.

\section{References}

1. Childs, P.R.N.; Greenwood, J.R.; Long, C.A. Review of temperature measurement. Rev. Sci. Instrum. 2000, 71, doi:10.1063/1.1305516.

2. Weinert, F.M.; Kraus, J.A.; Franosch, T.; Braun, D. Microscale fluid flow induced by thermoviscous expansion along a traveling wave. Phys. Rev. Lett. 2008, 100, doi:10.1103/ PhysRevLett.100.164501.

3. Homola, J.; Yee, S.S.; Gauglitz, G. Surface plasmon resonance sensors: Review. Sens. Actuators $B$ 1999, 54, 3-15.

4. Yager, P.; Edwards, T.; Fu, E.; Helton, K.; Nelson, K.; Tam, M.R.; Weigl, B.H. Microfluidic diagnostic technologies for global public health. Nature 2006, 442, 412-418.

5. Ross, D.; Gaitan, M.; Locascio, L.E. Temperature measurement in microfluidic system using a temperature-dependent fluorescent dye. Anal. Chem. 2001, 73, 4117-4123.

6. Irace, A.; Breglio, G. All-silicon optical temperature sensor based on multi-mode interference. Opt. Express 2003, 11, 2807-2812.

7. Wu, Y.; Rao, Y.J.; Chen, Y.H.; Gong, Y. Miniature fiber-optic temperature sensors based on silica/polymer microfiber knot resonators. Opt. Express 2009, 20, 18142-18147.

8. Dong, C.H.; He, L.; Xiao, Y.F.; Gaddam, V.R.; Ozdemir, S.K.; Han, Z.F.; Guo, G.C.; Yang, L. Fabrication of high-Q polydimethylsiloxane optical microspheres for thermal sensing. Appl. Phys. Lett. 2009, 94, doi:10.1063/1.3152791.

9. Ma, Q.L; Rossmann, T.; Guo, Z.X. Whispering-gallery mode silica microsensors for cryogenic to room temperature measurement. Meas. Sci. Technol. 2010, 21, doi:10.1088/0957-0233/21/2/025310.

10. Wang, X.P.; Yin, C.; Sun, J.J.; Li, H.G.; Wang, Y.; Ran, M.W.; Cao, Z.Q. High-sensitivity temperature sensor using the ultrahigh order mode-enhanced Goos-Hänchen effect. Opt. Express 2013, 21, 13380-13385.

11. Ozdemir, S.K.; Turban-Sayan, G. Temperature effects on surface plasmon resonance: Design considerations for an optical temperature sensor. J. Lightwave Technol. 2003, 21, 805-814.

12. Chiang, H.-P.; Yeh, H.T.; Chen, C.M.; Wu, J.C.; Su, S.Y.; Chang, R.; Wu, Y.J.; Tsai, D.P.; Jen, S.U.; Leung, P.T. Surface plasmon resonance monitoring of temperature via phase measurement. Opt. Commun. 2004, 241, 409-418.

13. Davis, L.J.; Deutsch, M. Surface plasmon based thermo-optic and temperature sensor for microfluidic thermometry. Rev. Sci. Instrum. 2010, 81, doi:10.1063/1.3499238.

14. Homola, J. Surface plasmon resonance sensors for detection of chemical and biological species. Chem. Rev. 2008, 108, 462-492.

15. Sun, Z.L.; He, Y.H.; Guo, J.H. Surface plasmon resonance sensor based on polarization interferometry and angle modulation. Appl. Opt. 2006, 45, 3071-3076. 
16. Liu, Z.Y.; Yang, L.; Liu, L.; Chong, X.Y.; Guo, J.; Ma, S.H.; Ji, Y.H.; He, Y.H. Parallel-scan based microarray imager capable of simultaneous surface plasmon resonance and hyperspectral fluorescence imaging. Biosens. Bioelectron. 2011, 30, 180-187.

17. Zhang, P.F.; Liu, L.; He, Y.H.; Shen, Z.Y.; Guo, J.; Ji, Y.H.; Ma, H. Non-scan and real-time multichannel angular surface plasmon resonance imaging method. Appl. Opt. 2014, 53, 6037-6042.

18. Shankar, P.; Viswanathan, N.K. All-optical thermo-plasmonic device. Appl. Opt. 2011, 50, 5966-5969.

19. Chiang, H.-P.; Chen, C.-W.; Wu, J.-J.; Li, H.-L.; Lin, T.-Y.; Sanchez, E.-J.; Leung, P.-T. Effects of temperature on the surface plasmon resonance at a metal-semiconductor interface. Thin Solid Films 2007, 515, 6953-6961.

20. Moreira, C.S.; Lima, A.M.N.; Neff, H.; Thirstrup, C. Temperature-dependent sensitivity of surface plasmon resonance sensors at the gold-water interface. Sens. Actuators B 2008, 134, 854-862.

21. Sharma, A.K.; Pattanaik, H.S.; Mohr, J.G. On the temperature sensing capability of a fibre optic SPR mechanism based on bimetallic alloy nanoparticles. J. Phys. D Appl. Phys. 2009, 42, doi:10.1088/0022-3727/42/4/045104.

22. Kim, I.T.; Kihm, K.D. Full-field and real-time surface plasmon resonance imaging thermometry. Opt. Lett. 2007, 32, 3456-3458.

23. Shao, L.; Shevchenko, Y.; Albert, J. Intrinsic temperature sensitivity of titled fiber Bragg grating based surface plasmon resonance sensors. Opt. Express 2010, 18, 11464-11471.

24. Zhang, P.F.; Liu, L.; He, Y.H.; Xu, Z.H.; Ji, Y.H.; Ma, H. One-dimensional angular surface plasmon resonance imaging based array thermometer. Sens. Actuators B 2015, 207, 254-261.

25. Liu, L.; Ma, S.H.; Ji, Y.H.; Chong, X.Y.; Liu, Z.Y.; He, Y.H.; Guo, J.H. A two-dimensional polarization interferometry based parallel scan angular surface plasmon resonance biosensor. Rev. Sci. Instrum. 2011, 82, doi:10.1063/1.3553028.

26. Lahav, A.; Auslender, M.; Abdulhalim, I. Sensitivity enhancement of guided-wave surface-plasmon resonance sensors. Opt. Lett. 2008, 33, 2539-2541.

27. Zhou, Y.F.; Zhang, P.F.; He, Y.H.; Xu, Z.H.; Liu, L.; Ji, Y.H.; Ma, H. Plasmon waveguide resonance sensor using Au-MgF2 structure. Appl. Opt. 2014, 53, 6344-6350.

28. Chien, F.C.; Chen, S.J. A sensitivity comparison of optical biosensors based on four different surface plasmon resonance modes. Biosens. Bioelectron. 2004, 20, 633-642.

29. Bahrami, F.; Maisonneuve, M.; Meunier, M.; Aitchison, J.S.; Mojahedi, M. An improved refractive index sensor based on genetic optimization of plasmon waveguide resonance. Opt. Express 2013, 21, doi:10.1364/OE.21.020863.

30. Szunerits, S.; Boukherroub, R. Electrochemical investigation of gold/silica thin film interfaces for electrochemical surface plasmon resonance studies. Electrochem. Commun. 2006, 8, 439-444.

31. Kretschmann, E.; Raether, H. Radiative decay of nonradiative surface plasmons excited by light. Z. Naturforsch. A 1968, 23, 2135-2136.

32. Bahrami, F.; Maisonneuve, M.; Meunier, M.; Aitchison, J.S.; Mojahedi, M. Self-referenced spectroscopy using plasmon waveguide resonance biosensor. Biomed. Opt. Express 2014, 5, 2481-2487.

33. Heavens, O.S. Optical properties of thin films. Rep. Prog. Phys. 1960, 23, 1-65. 
34. Born, M.; Wolf, E. Principles of Optics: Electromagnetic Theory of Propagation, Interference and Diffraction of Light, 7th ed.; Cambridge University: Cambridge, UK, 1999.

35. Shi, H.; Liu, Z.Y.; Wang, X.X.; Guo, J.; Liu, L.; Luo, L.; Guo, J.H.; Ma, H.; Sun, S.Q.; He, Y.H. A symmetrical optical waveguide based surface plasmon resonance biosensing system. Sens. Actuators B 2013, 185, 91-96.

36. Hansen, W.N. Electric fields produced by the propagation of plane coherent electromagnetic radiation in a stratified medium. J. Opt. Soc. Am. 1968, 58, 380-390.

37. Nenninger, G.G.; Tobiška, P.; Homola, J.; Yee, S.S. Long-range surface plasmons for high-resolution surface plasmon resonance sensors. Sens. Actuators B 2001, 74, 145-151.

38. Purcell, E.M.; Morin, D.J. Electricity and Magnetism, 3rd ed.; Cambridge University: Cambridge, UK, 2013.

39. Feynman, R.P.; Leighton, R.B.; Sands, M. The Feynman Lectures on Physics; Addison-Wesley: Boston, MA, USA, 1963.

40. Kittel, C.; McEuen, P. Introduction to Solid State Physics; Wiley: New York, NY, USA, 1976.

41. Schiebener, P.; Straub, J.; Sengers, J.M.H.L.; Gallagher, J.S. Refractive index of water and steam as function of wavelength, temperature and density. J. Phys. Chem. Ref. Data 1990, 19, 677-717.

42. Chiang, H.-P.; Leung, P.-T.; Tse, W.-S. Remarks on the substrate-temperature dependence of surface-enhanced Raman scattering. J. Phys. Chem. B 2000, 104, 2348-2350.

43. McKay, J.A.; Rayne, J.A. Temperature dependence of the infrared absorptivity of the noble metals. Phys. Rev. B 1976, 13, 673-684.

44. Beach, R.T.; Christy, R.W. Electron-electron scattering in the intraband optical conductivity of $\mathrm{Cu}$, Ag, and Au. Phys. Rev. B 1977, 16, 5277-5284.

45. Ballard, S.S.; Browder, J.S. Thermal expansion and other physical properties of the newer infrared-transmitting optical materials. Appl. Opt. 1966, 5, 1873-1876.

46. Weber, M.J. Handbook of Optical Materials; CRC Press: Boca Raton, FL, USA, 2003.

47. Dodge, M.J. Refractive properties of magnesium fluoride. Appl. Opt. 1984, 23, 1980-1985.

48. Browder, J.S.; Ballard, S.S. Thermal expansion data for eight optical materials from $60 \mathrm{~K}$ to $300 \mathrm{~K}$. Appl. Opt. 1977, 16, 3214-3217.

49. Grassi, J.H.; Georgiadis, R.M. Temperature-dependent refractive index determination from critical angle measurements: Implications for quantitative SPR sensing. Anal. Chem. 1999, 71, 4392-4396.

50. Chong, X.Y.; Liu, L.; Liu, Z.Y.; Ma, S.H.; Guo, J.; Ji, Y.H.; He, Y.H. Detect the hybridization of single-stranded DNA by parallel scan spectral surface plasmon resonance imaging. Plasmonics 2013, 8, 1185-1191.

51. Bahrami, F.; Alam, M.Z.; Aitchison J.S.; Mojahedi, M. Dual polarization measurements in the hybrid plasmonic biosensors. Plasmonics 2013, 8, 465-473.

52. Piliarik, M.; Homola, J. Surface plasmon resonance (SPR) sensors: Approaching their limits? Opt. Express 2009, 17, 16505-16517.

(C) 2015 by the authors; licensee MDPI, Basel, Switzerland. This article is an open access article distributed under the terms and conditions of the Creative Commons Attribution license (http://creativecommons.org/licenses/by/4.0/). 